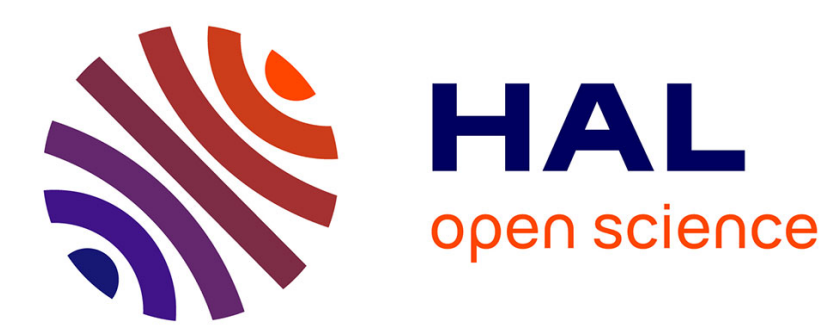

\title{
Structural studies of ionically conductive glasses by EXAFS
}

\author{
F. Rocca
}

\section{To cite this version:}

F. Rocca. Structural studies of ionically conductive glasses by EXAFS. Journal de Physique IV Proceedings, 1992, 02 (C2), pp.C2-97-C2-106. 10.1051/jp4:1992210 . jpa-00251281

\section{HAL Id: jpa-00251281 https://hal.science/jpa-00251281}

Submitted on 1 Jan 1992

HAL is a multi-disciplinary open access archive for the deposit and dissemination of scientific research documents, whether they are published or not. The documents may come from teaching and research institutions in France or abroad, or from public or private research centers.
L'archive ouverte pluridisciplinaire HAL, est destinée au dépôt et à la diffusion de documents scientifiques de niveau recherche, publiés ou non, émanant des établissements d'enseignement et de recherche français ou étrangers, des laboratoires publics ou privés. 


\title{
Structural studies of ionically conductive glasses by EXAFS
}

\section{F. ROCCA}

Centro CNR-ITC di Fisica degli stati aggregati ed impianto ionico, Via Sommarive 14, 38050 Povo (Trento), Italy

\begin{abstract}
$\underline{\text { Abstract }}$
A good knowledge of the local structure is necessary for a better understanding of the high conductivity shown by some ions in oxide glasses.

The ability of X-Ray Absorption Spectroscopy (XAS) to select a single atomic species makes this technique very useful in this field: XANES and EXAFS can give information not only on radial distribution function of atoms around the absorbing species but also on their geometrical arrangement.

The experience of the last years shows that peculiar information can be extracted by studying multiple-scattering contributions and evaluating the influence of static and thermal disorder.

The results of XAS studies on AgI-containing silver borate glasses are presented and discussed, with particular emphasis on the role of temperature dependent measurements in EXAFS.

The comparison with results concerning other similar systems offers the possibility of discussing strengths and limits of XAS.
\end{abstract}

\section{INTRODUCTION}

The availability of X-Ray Synchrotron radiation sources has allowed the great development of X-Ray Absorption Spectroscopy (XAS) during the last 15 years./1/ By selecting different absorption edges, the study of the local environment of single atomic species can be easily performed. This is particularly interesting for amorphous compounds, because the absence of long range order decreases the sensitivity of usual diffraction methods. In particular, EXAFS (Extended X-Ray Absorption Fine Structure) contains important local structural information like interatomic distances, coordination numbers and mean square relative displacements.

A standard analysis of EXAFS data can easily show the presence of short range order in disordered materials; the improvement in methods of analysis done during the last years let EXAFS be able to face more complicated and difficult problems, like the characterization of non gaussian distribution functions of distances or the study of the local vibrational dynamics. These studies need a careful approach, since the limits of the simple one-electron single scattering theory are well defined and the problems related to static or thermal disorder require appropriate procedures./2-3/

The major improvement in XAS of the last years is the development of theories and related software packages able to calculate ab initio an absorption spectrum, taking into account both 
single and multiple scattering effects./4-5/ This offers the possibility of simulating the local environment and calculating an EXAFS or XANES spectrum to be compared directly with the experimental one. In this way XAS can give peculiar information not only on distances, but also on relative positions of atoms around the absorbing species.

This paper presents some exemplary results obtained on oxide glasses, to emphasize the peculiar information that XAS has provided in the characterization of these systems. In particular, the fast ion conducting glasses $\mathrm{AgI}: \mathrm{Ag}_{2} \mathrm{O}: \mathrm{B}_{2} \mathrm{O}_{3}$ have been studied by XAS, aiming to have a better knowledge of the local structure of these systems and to improve the understanding of the high ionic conduction of glasses./6/ The results of these studies are presented here and reviewed on the grounds of our latest temperature dependent measurements and of comparative results obtained with other techniques or for similar systems.

A short introduction to XAS fundamentals is preliminarly given in order to clarify the crucial points of theory and to emphasize strengths and limits of this experimental technique.

\section{FUNDAMENTAL OF XAS}

A XAS spectrum can be divided in at least three regions, where different physical processes are predominant: the edge region near the absorption threshold $E_{0}$, the XANES region in the range $10-40 \mathrm{eV}$ beyond $\mathrm{E}_{0}$ and then the EXAFS region. This classification is very useful for a general understanding of the physical phenomena involved, but it should be noted that the energy limits between different parts of an absorption spectrum are only marked as reference for this discussion. In the edge region, the main contribution to the change in the absorption coefficient derives from the density of empty electronic states that can be filled by a direct transition from a core level. A band structure formalism or a molecular orbital description of electronic density around the absorbing atom are able to describe the structures in the edge region. This approach allows the direct comparison of spectra related to different compounds, and has been shown to be very useful in the study of coordination chemistry, where the change in valence and/or in coordination number is the most important information to be extracted from a XAS spectrum. In a few cases, the spectrum exhibits a sharp absorption line in the proximity of the main absorption edge, usually called "white line": this indicates the presence of unoccupied localized electronic levels and is very sensitive to changes in the chemical bond.

The main difference between XANES and EXAFS is the role of multiple scattering processes of the photoelectrons, that are predominant in the XANES region and become less and less efficient at higher energies. The evaluation of single and higher order scattering terms has been done for some simple compounds during the last years and now it is well clear that some configurations (for example a linear coordination between three atoms) can significantly contribute also in the classical EXAFS region./7/

Since the calculation of multiple scattering contributions requires a deep knowledge of theory and long computer-time, only a few studies have been published on this subject: a significant improvement to XAS is expected for the next years from studies of higher order correlations functions.

The single-scattering contribution to EXAFS oscillations is described by :

$$
\chi(k)=\sum_{j} \frac{S_{0}^{2} N_{j} f_{j}(k, \pi)}{k R_{j}^{2}} e^{-2 R_{j} / \lambda} e^{-2 \sigma_{j}^{2}(T) k^{2}} \sin \left(2 k R_{j}+\phi_{j}\right)
$$

where $\phi_{\mathrm{j}}(\mathrm{k})$ is the total phaseshift function containing contributions of backscattering and 
absorbing atoms; $f_{j}(k, \pi)$ is the backscattering amplitude function; $\lambda(k)$ is the mean-free path of photoelectron; $\mathrm{S}_{0}{ }^{2}$ and $\lambda(\mathrm{k})$ take into account the inelastic losses of photoelectrons.

The second exponential term is a Debye-Waller-like factor (D.W.), where $\sigma_{j}^{2}$ is the meansquare relative displacement (MSRD) of atoms along the bond direction./8/ Eq.1 contains a sum over all possible (and experimentally detectable) coordination shells around a selected atomic species in the system. In a harmonic crystal, each shell is described by a gaussian distribution function of atoms vibrating at the crystallographic positions. The behaviour of $\sigma_{j}^{2}$ has been shown very sensitive to the correlation of motion of atom pairs and contains peculiar information on the polarization of phonons./9/ At increasing temperature, the anharmonicity becomes important and a simple gaussian distribution function is no longer a correct description of the real coordination./10/

Many errors in EXAFS data analysis come from an underestimate of the effects of a non gaussian distribution function of interatomic distances: this can produce a wrong determination not only of coordination numbers but also of mean distances./2-3/ This problem is crucial in the study of amorphous systems, because the lack of long range order and different local strains can produce a static spread of interatomic distances. However, the experience of the last years shows that this is not always true: some atoms may have a more ordered local coordination in glasses than in the corresponding crystalline compounds, as we will see later.

The usual analysis of an EXAFS signal begins by singling out a one-shell contribution from the total $\chi(k)$ function. After this, a quantitative evaluation can be performed by using the backscattering amplitude and phaseshifts functions of the atom pair under study. If good experimental EXAFS signals of some reference compounds are available, they can be used to evaluate coordination numbers, distances and relative mean square displacements of the unknown sample. This procedure requires special care, because the hypothesis of transferability of amplitude and phaseshifts functions must be checked each time. The comparative analysis between the functions of the unknown and reference compounds is very useful to extract structural information for moderate disorder. These methods allow to analyze independently phase and amplitude functions and can easily emphasize the presence of a non gaussian distribution of distances in a selected coordination shell. The lower number of free parameters used in calculating either distances or coordination numbers and MSRDs respectively, improves the reliability of quantitative analysis .

If this simple procedure was unable to determine accurately the physical quantities, the usual best fit methods based on the superposition of two o more gaussian shells should be used with great attention: even if the final best fit is good, the correlation between free parameters might give wrong physical results.

Alternatively, one can begin by modeling a partial distribution function of distances $P(r)$. This function can be used for a comparison in the R-space with the Fourier transformed EXAFS signal. Only a few works based on this method have been published, but the obtained results are very important in the short history of EXAFS (see for example the papers of Boyce and Hayes on AgI and similar compounds /11/).

A further possibility is to calculate the first terms of the cumulant expansion series of $\mathrm{P}(\mathrm{r}) . / 12$ The same terms can be extracted from the EXAFS signal by a careful analysis of amplitudes and phases, at least in the range of non very high disorder. The reliability of this procedure in presence of an asymmetric distribution function of distances depends both on the value of cumulants and on the shape of distribution: some results obtained with this method are shown in this volume./13/

At present, it is also possible to calculate the phaseshifts and amplitude functions ab initio, and later to insert them in the calculation of total EXAFS signal /4-5/: this allows the best fitting of EXAFS directly in the k-space, starting from a model of the distance distribution functions. In this way we are no more forced to evaluate the single shell contributions once a time, and the EXAFS experimental signal can be analysed as people is used to do for diffraction experiments. 


\section{XAS OF BINARY OXIDE GLASSES}

\subsection{The glass network structure.}

The easiness of changing the network matrix of oxide glasses allows the insertion of alkali oxide in a wide range of concentrations. The glass network is modified by this mixing in order to maintain charge and chemical equilibrium, and to provide a suitable volume for the insertion of different atoms. This process is far to be understood: its complexity needs the convergence of different experimental and theoretical approaches.

The contribution of XAS in this field has been decisive in developing a realistic structural model for glasses containing modifier oxides. In fact, the Zachariasen model /14/ called Continuous Random Network (CRN) was based only on network former units: the modifying cations were allowed to be randomly distributed in the glass, without affecting the ordering of the structural units of the network. The short range information derived by XAS measurements forced to develop new models. The evidence of well defined local coordination for $\mathrm{Ca}$ and $\mathrm{Na}$ cations in silicate glasses was shown for the first time from EXAFS by Greaves et al. /15/. The Modified Random Network model (MRN), developed after the EXAFS measurements on silicate glasses, assumes that the modifying cations may be bonded to non-bridging oxygens (NBO) in some modified regions of the network, where they can have a higher mobility./16/ These regions act like percolation pathways, having an extension dependent on concentration. The local environment of cations changes significantly as a function of composition when two different cations are inserted in glasses. The obtained XAS results constitute a structural basis for a better understanding of the mixed-alkali effect. The relationship between ion conductivity and structure, both local and intermediate, is at present under study: some published results seem very promising, at least for silicate glasses./17/

An important information provided by EXAFS besides interatomic distances and coordination numbers concerns the static distortion measured in the nearest and next nearest neighbour coordination shells of network former atoms. This information is usually obtained by comparing the D.W. factors of glassy and crystalline compounds at the same temperature.

The D.W. factor of the first coordination shell (e.g. the Si-O bond in silicate glasses) has been shown to be rather low, sometimes lower in glasses than in crystals. The ability of EXAFS in detecting the presence of short range order in disordered systems is not overcome by other experimental techniques.

A different problem is the quantitative analysis of "disorder" or "distortion" in the intermediate range. The low electron mean free path and the loss of information in the low $\mathbf{k}$ range of the EXAFS function are the main differences in respect with diffraction measurements: the longer the distance, the more difficult is the analysis of contributions of next nearest neighbour shells to total EXAFS signal. However, the analysis of D.W. factors of the second coordination shell of amorphous $\mathrm{GeO}_{2}$ allowed for the first time an estimate of the angular distortion of Ge-O-Ge bonds already in 1975./18/ The first EXAFS experiments in this field appeared very promising, but the refinement of analysis in the following years pointed out the limits of a standard method./19/ Many parameters influence the reliability of an analysis of second nearest neighbours in glasses and high quality experimental data are requested. Temperature dependent measurements must be performed in order to distinguish between static and thermal disorder, when the main contribution to D.W. factor can be attributed to a single shell coordination. If chemical disorder is present, only very sophisticated strategies can be used for the evaluation of structural parameters./20/

A more promising approach is the calculation of multiple scattering contributions, that contain peculiar information on the relative positions of atoms around the absorbing species. Since at low $\mathbf{k}$ the electron mean free path is longer, the XANES region is influenced also by atoms in the outer neighbour shells. The studies on amorphous $\mathrm{SiO}_{2}$ performed with this method are exemplary: the XAS spectrum has been calculated for different Si-O-Si and O-Si-O angles in a small cluster of atoms./21/ Changes in the bonding angles produce significantly different spectra: the comparison of experimental spectra with the calculated ones allows to develop more realistic models and to take into account variations due, for example, to different thermal history 
or pressure effects.

By multiple scattering calculations it will be possible to study more carefully the changes in the region beyond the first coordination shell when some modifier oxides are mixed with a glass former compound. The possibility of modifying the charge equilibrium around the atoms of silicate or phosphate glass networks leads to very different situations, because the number on NBOs and the relative linkage of tetrahedral basic structural units change with composition. The ionic conductivity of these glasses is also changing as a function of composition and of the kind of cations inserted in the glass: a deep knowledge of the local structure is still lacking. The XAS spectra of some phosphate compounds (crystalline and glassy) in Fig. 1 clearly show the sensitivity of this technique. Note that the edge structures are influenced also by the substitution of cations: for the first $10 \mathrm{eV}$, this is mainly related to a modification of electronic levels of phosphorus atoms due to the presence of different cations. On the contrary, EXAFS studies of some silver phosphate glasses at the phosphorous edge were not able to identify the second coordination shell./22/

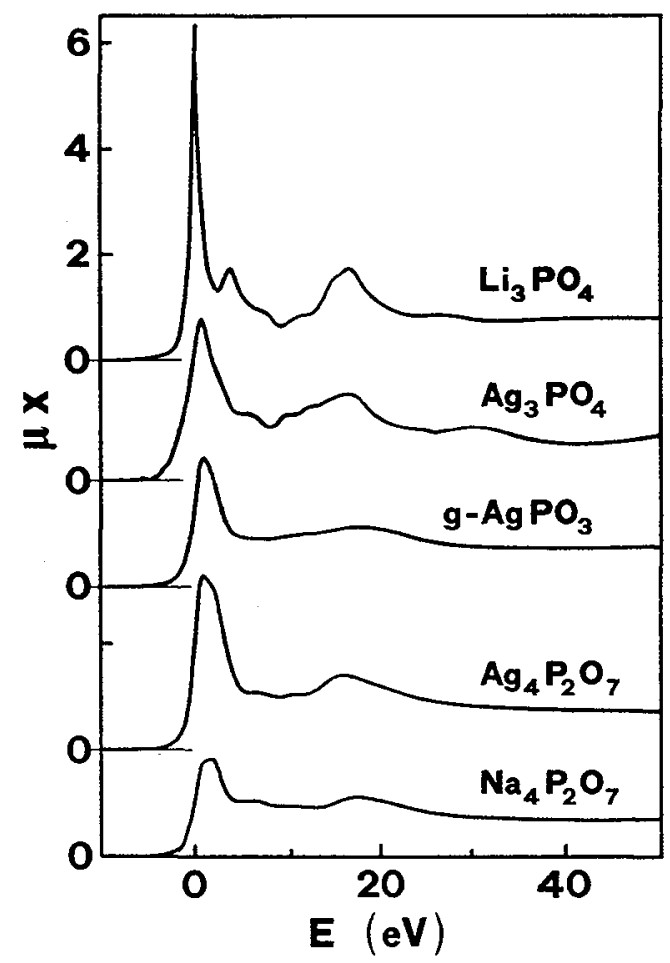

FIGURE 1: XANES spectra at the Phosphorous K-edge. Measurements performed at LURE - SUPERACO (Orsay -France).

For a qualitative comparison, spectra have been normalized at $50 \mathrm{eV}$ after the edge energy.

\subsection{The cation environment in borate glasses}

The case of borate network is completely different from that of silicate and phosphate glasses. In fact, beside $\mathrm{BO}_{4}$ tetrahedral units, $\mathrm{BO}_{3}$ planar units are present in these glasses. These two basic units can link together to form the larger structural groups (classified by Krogh-Moe /23/) characteristic of borate glasses. This more free rearrangement of network units leads to a different presence of NBOs with respect to silicate glasses: NBOs are absent in the original $\mathrm{B}_{2} \mathrm{O}_{3}$ glass and up to a concentration ratio $1: 2$ between modifying oxide and $\mathrm{B}_{2} \mathrm{O}_{3}$. The information about NBOs comes from many NMR studies on borate glasses, $/ 24$ / (boron is out of the possibility of EXAFS) and forces to model the local structure around the cations with some differences in respect with silicate glasses.

In fact, if the covalent bond provided by NBOs is not present, the modifying cations should be coordinated to a higher number of oxygens, as expected by the continuum random network model. EXAFS measurements, however, show that the role of different cations may be very 
different: the study of sodium and silver environment in borate glasses clearly indicates that a unique model for the structure of these glasses is unrealistic.

Room temperature EXAFS measurements at the Na-K edge of the glass $\mathrm{Na}_{2} \mathrm{~B}_{2} \mathrm{O}_{7}$ were performed by Greaves et al./25/ in one of the first EXAFS study on glasses. Their analysis showed about 6 oxygen atoms around $\mathrm{Na}$, and a very high D.W. factor. This value is significantly higher than that measured by the same authors for the $\mathrm{Na}$ environment in sodium silicate glasses and was interpreted as an indication of a high static disorder.

The study of silver environment in borate glasses has been done at the Ag-K and - L edges at room temperature./26-27/ For the analysis of L edges, we compared the XANES structure of $c-\mathrm{Ag}_{2} \mathrm{O}$ and of glasses. The XANES region at $\mathrm{L}_{3}$ edge of $\mathrm{Ag}_{2} \mathrm{O}(3.351 \mathrm{KeV})$ is very structured, and shows a characteristic white line. This feature has been attributed to a direct transition of the photoelectron from the $2 \mathrm{p}$ core level to the $\mathrm{Ag}-4 \mathrm{~d}$ partial density of electronic states./28/ This band is filled for about $90 \%$; the unoccupied states are present at the bottom of conduction band in a small region, thus contributing to the first peak of the absorption spectrum. In $c-\mathrm{Ag}_{2} \mathrm{O}$ the silver atoms are linearly coordinated to two oxygen atoms, at a distance $2.044 \AA$. This low coordination is rather unusual; the presence of the same peak in the XANES spectra of $\mathrm{Ag}_{2} \mathrm{O} \mathrm{n} \mathrm{B}_{2} \mathrm{O}_{3}$ glasses $(\mathrm{n}=2,3,4,6)$ has been interpreted as an indication that the same kind of bond is still present in the glasses. The low coordination number was confirmed by EXAFS measurements, the Ag-O bond length was larger in glasses, about $2.3 \AA$. The best-fit of filtered EXAFS spectra was performed by using the experimental backscattering phaseshifts and amplitude of $\mathrm{c}-\mathrm{Ag}_{2} \mathrm{O}$ and showed for the glasses a D.W. factor comparable with that of crystalline model compound at room temperature. Following these XAS results and taking into account the absence of NBOs for the glasses with $n>2$, we developed a simple model for the silver coordination in these glasses: the low D.W. factor was explained by a bonding of silver to oxygens belonging to the same $\mathrm{BO}_{4}$ tetrahedron. Recently, we found some evidence against this simple model. An analysis of diffraction spectra of silver and lithium diborate glasses,/29/ shows the existence of differences in the intermediate range order due to the change of lithium with silver atoms. Silver is more probably bridging between different Krogh-Moe units of the matrix. This means that silver is playing an active role in fixing the relative linkage of different units in the glass. In this contest the low D.W. factor measured at room temperature is rather surprising; on the contrary, some preliminary results coming from low temperature measurements at the Ag-K edge show that the mean square relative displacements of the $\mathrm{O}-\mathrm{Ag}-\mathrm{O}$ distances in $\mathrm{Ag}_{2} \mathrm{O}$ and in the glasses are different. The analysis by the ratio method of $\mathrm{Ag}$ coordination at temperature $50 \mathrm{~K}$ in the glass $\mathrm{Ag}_{2} \mathrm{O}_{4} \mathrm{~B}_{2} \mathrm{O}_{3}$ confirms the low coordination number, but shows a $\mathrm{D}$.W. factor for the $\mathrm{Ag}-\mathrm{O}$ bond in the glass significantly higher than in $\mathrm{c}-\mathrm{Ag}_{2} \mathrm{O}$.

The low coordination number seems to be typical of silver and differentiates this cation from all the others modifiers in glasses.

Recent temperature measurements at Ag-K edge in Ag-As-S and Ag-As-Se glasses confirm this fact:/30/ silver is coordinated by two sulphur (selenium) atoms, whatever the silver composition of glasses. A well defined second shell peak is present in the radial distribution function obtained by EXAFS at low temperature, indicating a rather ordered structure around silver atoms.

The insertion of silver by ion exchange with $\mathrm{Na}$ in sodium alumino silicate glasses has been studied by EXAFS and once more shows the difference between these cations: silver is not simply substituting sodium, but its coordination "compare closely to the immediate environment of $\mathrm{Ag}$ in crystalline $\mathrm{Ag}_{2} \mathrm{O}^{\prime \prime} . / 31 /$

Coming back to the problem of silver ion conductivity in borate glasses, a structural model must accommodate the evidence of a low coordination in a quasilinear configuration with the claimed absence of NBOs, at least up to a $33 \%$ mol. content of $\mathrm{Ag}_{2} \mathrm{O}$. This is very difficult, because the Ag atoms seem to be strongly bonded to the oxygens of the glass network, independently of composition.

Further low temperature XAS measurements on glasses with different $\mathrm{Ag}_{2} \mathrm{O}$ content are planned in order to study the influence of NBOs on the coordination of $\mathrm{Ag}$. We are studying also 
the shape of the partial distribution function of the Ag-O distances as a function of temperature: the comparison between $\mathrm{c}-\mathrm{Ag}_{2} \mathrm{O}$ and glasses should lead to an evaluation of static and thermal contributions to the relative displacements of atoms in the first coordination shell.

It is most likely that the Ag-O bond length changes for bridging and non-bridging oxygens, thus modifying the distribution function and the connected vibrational dynamics. A recent molecular dynamics study of sodium silicate glasses confirms the different role of bridging and non-bridging oxygens in determining the distribution function of $\mathrm{Na}-\mathrm{O}$ distances./32/ It is evident from this study that the Na-O peak corresponding to bonds with NBOs is narrower and the mean distance slightly shorter. We can anticipate that it would be rather difficult to study a similar environment for silver by EXAFS with the standard analysis methods: however we trust that measurements as a function of temperature will help in understanding the experiments.

\section{XAS OF SILVER BORATE GLASSES DOPED WITH SILVER IODIDE}

The insertion of $\mathrm{AgI}$ in the matrix $\mathrm{Ag}_{2} \mathrm{O} \cap \mathrm{B}_{2} \mathrm{O}_{3}$ greatly enhances the $\mathrm{Ag}^{+}$conductivity. XAS studied on $\mathrm{Ag}$ and $\mathrm{I}$ edges have been performed as a function of composition and of temperature./33-34/ There are two main open problems: the first concerns the characterization of the coordination of $\mathrm{Ag}^{+}$ions with oxygen and iodine atoms; the second concerns the possibility, suggested by some authors, of having in the glass a crystal-like structure for AgI.

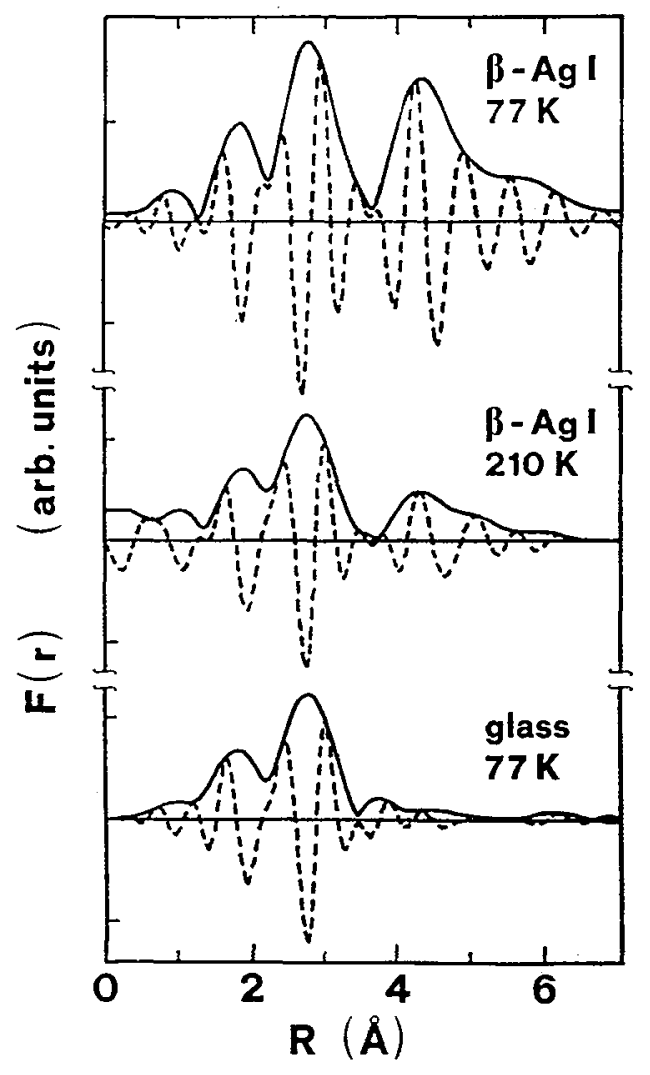

FIGURE 2: Modulus (continuous line) and imaginary part (dashed line) of the Fourier transform of $I_{3}$ EXAFS function of $B$-AgI measured at $77 \mathrm{~K}$ and $210 \mathrm{~K}$ and of glass $(\mathrm{AgI})_{0.55}\left(\mathrm{Ag}_{2} \mathrm{O} 4 \mathrm{~B}_{2} \mathrm{O}_{3}\right)_{0.45}$ at $77 \mathrm{~K}$. Measurements performed at ADONEPWA (Frascati - Italy)

Our studies of Ag-L edge structures show that the O-Ag-O coordination typical of the binary matrix persists in the AgI containing glasses. The XANES spectra can be best-fitted by a 
linear combination of the experimental spectra of $\mathrm{Ag}_{2} \mathrm{O} \mathrm{n} \mathrm{B}_{2} \mathrm{O}_{3}$ and $\mathrm{B}-\mathrm{AgI}$, following the stoichiometry ratio./6/ This means that the bond of silver with the oxygens of the borate network is not appreciably affected by the progressive introduction of AgI. Moreover EXAFS analysis shows the presence of two distinct coordinations around silver: $\mathrm{Ag}-\mathrm{O}$ and $\mathrm{Ag}-\mathrm{I}$. A quantitative analysis as a function of composition is still lacking, due to the low quality of our EXAFS signals at room temperature.

The most important result we have obtained concerns the local structure around iodine atoms. The first coordination shell of iodine in the glasses looks like that of B-AgI, whatever the composition. The coordination number is the same for AgI and the glasses; a careful analysis shows the presence of asymmetry in the pair distribution function of I-Ag distances. Furthermore, the next neighbour coordination shells are very different, and change as a function of

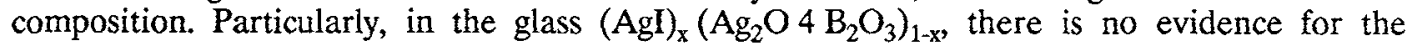
iodine-iodine coordination of the second shell of crystalline AgI. On the contrary, at low AgI content there is evidence of an I-Ag-O coordination. The corresponding peak progressively disappears from the Fourier filtered spectrum at increasing the AgI concentration. At low AgI content, the local arrangement around iodine seems to be more ordered: iodine ions are quite dispersed but they are all bonded to silver atoms interfacing with the glass network. This local arrangement is likely present also at higher $\mathrm{AgI}$ content, but the predominant contribution to EXAFS signal becomes that of iodine atoms inside the AgI-rich regions. The absence from EXAFS signal of the contribution of the iodine-iodine coordination in the glass $(\mathrm{AgI})_{0.55}\left(\mathrm{Ag}_{2} \mathrm{O} 4 \mathrm{~B}_{2} \mathrm{O}_{3}\right)_{0.45}$ has been confirmed by measurements at $77 \mathrm{~K} . / 34 /$ At low temperature the thermal contribution to D.W. factor is strongly reduced, as shown in fig.2 for cAgI. In summary, EXAFS reveals a high static disorder around iodine atoms in the glass, and excludes the presence of medium-range ordered clusters of silver iodide.

FIGURE 3: EXAFS Debye-Waller

factors measured at the Iodine $\mathrm{L}_{3}$ edge of $\mathrm{B}-\mathrm{AgI}$ (full circles) and glass

$(\mathrm{AgI})_{0.55}\left(\mathrm{Ag}_{2} \mathrm{O} 4 \mathrm{~B}_{2} \mathrm{O}_{3}\right)_{0.45}$ (empty circles). The line represents the Einstein model with frequency $2.65 \mathrm{THz}$.

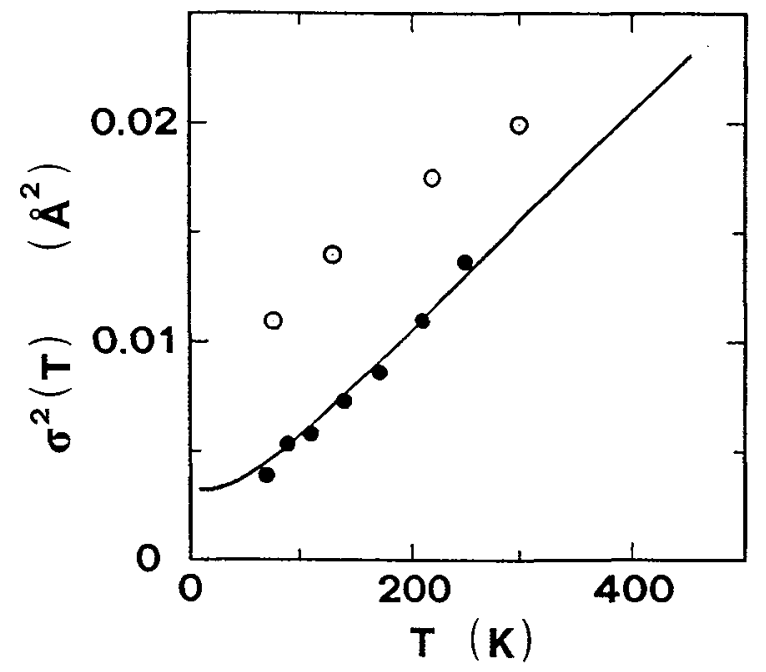

We have been able to quantify the degree of disorder by an analysis of the first coordination shell of iodine as a function of temperature. To reach this goal, we previously characterized the I-Ag coordination of B-AgI as a function of temperature. The ratio method was applied to extract the first even cumulants of the distribution both for AgI and the glass $(\mathrm{AgI})_{0.55}\left(\mathrm{Ag}_{2} \mathrm{O}_{4} \mathrm{~B}_{2} \mathrm{O}_{3}\right)_{0.45}$. The second cumulant represents the change of the Debye-Waller factor in respect with that of the reference spectrum. The temperature behaviour of $D$.W. factor for the first coordination shell of the crystal has been best-fitted with simple Einstein model to calculate its absolute value./9/ Thus we could evaluate the D.W. factor of the glass and measure both static and thermal contribution. Already at low temperature the distribution function corresponding to I-Ag distance is characterized by a very high D.W. $\sigma^{2}=0.011 \AA^{2}$, more than 
twice than for $B-A g I$ at the same temperature. This difference is mainly due to the high static disorder present in the glass, while the $\mathrm{B}-\mathrm{AgI}$ at $77 \mathrm{~K}$ shows a gaussian distribution. By using the static D.W. factor, we can estimate a difference in bond lengths of the order of $\Delta R=0.15 \AA$ within the first I-Ag shell in the glass.

The temperature behaviour of D.W. factors is shown in Fig. 3, where the continuous curve was calculated by an Einstein frequency $2.65 \mathrm{THz}$. The same curve, upward shifted to consider the static contribution at $77 \mathrm{~K}$, can describe with reasonable approximation also the D.W. factor of glass, at least up to $220 \mathrm{~K}$, where the asymmetry is so high that the data analysis method can be less effective./13/

In spite of the very different conductivity and distance distribution function of the two compounds, the behaviour of the thermal contribution to D.W. factor indicates that the local vibrational dynamics is very similar for B-AgI and the measured glass. Further measurements on glasses with different composition and ionic conductivity are requested to confirm and better understand this similarity. The recent stabilization of superionic $\alpha$-AgI at room temperature in a glass matrix / 35 / offers the possibility to compare the thermal behaviour of I-Ag coordination directly with the fast-ionic conducting phase of $\mathrm{AgI}$ in a wide temperature range: this study is very promising for the comprehension of the high conductivity shown by $\mathrm{Ag}$ both in the high temperature phase of AgI and in AgI-containing silver borate glasses glasses.

\section{CONCLUSIONS}

The ability of XAS in studying the local environment of single atomic species in disordered materials has been shown very powerful, together with the possibility of characterizing nonsymmetric distance distribution functions. However, the study of disordered systems is often a very complicated problem, that needs the convergence of many experimental techniques. The comparison of results coming from different experimental techniques requires the previous evaluation of accuracy of single experiments. XAS gives careful information on short range order, and is able to study non-gaussian distribution functions of distances. At intermediate range, the multiple scattering analysis may provide information about the bond angles and the geometrical arrangement of atoms.

For a more accurate modeling of the whole structure of amorphous systems an effort should be done in the next years to extract the structural information by a combined analysis of many experiments, in particular XAS and diffraction methods which are often complementary. In this way, limits and strengths of each technique may be evaluated simultaneously and within the same mathematical constraints. The final result will reach the maximum of compatibility with the full set of experiments. A nonlinear multi-experiment approach has been proposed and tested by Blau et al./20/, obtaining a set of partial distribution functions. A different approach is the Reverse Montecarlo technique (RMC) /36/, that models a structural picture of an amorphous compound compatible with single or multiple experiments. The resulting partial distribution functions are a statistical average of all different local configurations calculated by the model.

The 3rd generation Synchrotron radiation sources will provide for the next years very intense and experiment tailored beamlines: an improvement of methods for the modeling of disorder materials is requested for a deeper understanding of new experiments.

\section{ACKNOWLEDGEMENT}

The research activity on AgI-containing glasses has been done in collaboration with G.Dalba and P.Fornasini (Dept. of Physics - Trento University), to whom the author is indebted and grateful. 


\section{REFERENCES}

1 Lee P.A., Citrin P.H., Eisenberger P. and Kinkaid B.M., Rev. Mod. Phys. 53 (1981) 769

2 Eisenberger P. and Brown G.S., Sol. State Comm. 29 (1979) 481

3 Crozier E.D., Rehr J.J. and Ingalls R., Amorphous and liquid systems, in X-Ray Absorption, ed. by D.C. Konongsberger and R. P rins, John Wiley \& Sosn, New York (1988) 373

4 Natoli C.R. and Benfatto M., J. Physique 47 C-8 (1986) 11

5 Rehr J.J., Albers R.C. and Mustre de Leon J., Phisica B 158 (1989) 417; Munstre de Leon J., Rehr J.J., Zabinski S.I. and Albers R.C., Phys.Rev. B44 (1991) 4146

6 Dalba G., Fornasini P. and Rocca F., J. Non-Cryst. Solids 123 (1990) 310

7 Benfatto M., Natoli C.R., Bianconi A., Garcia J., Marcelli A., Fanfoni M. and Davoli I, Phys. Rev. B34 (1986) 5774

8 Beni G. and Platzman P.M., Phys. Rev. B 14, (1976) 1514

9 Dalba G., Fornasini P., Rocca F. and Mobilio S., Phys. Rev. B 41 (1990) 9668

10 Tranquada J.M. and Ingalls R., Phys. Rev. B 28 (1983) 3520

11 Boyce J.B. and Hayes, "Structural studies of superionic conduction", in EXAFS Spectroscopy Techniques and Applications, ed. by B.K. Teo and D.C. Joy, Plenum, New York (1981) 84

12 Bunker G., Nucl. Instr. Method 207 (1983) 437

13 Dalba G., Fornasini P., Rocca F. and Sadoc A., J. de Physique C (1992) this volume

14 Zachariasen W.H., J. Am. Chem. Soc. 54 (1932) 3841

15 Greaves G.N., Fontaine A. Lagarde P., Raoux D. and Gurman S.J., Nature 293 (1981) 611

16 Greaves G.N., J. Non-Cryst. Solids 71 (1985) 203

17 Greaves G.N., Gurman S.J., Catlow C.R.A., Chadwick A.V. Houde-Walter S., Dobson B and Henderson C.M.B., Phil. Mag. 64 (1991) 1059

18 Sayers D.E., Stern E. and Lytle F.W., Phys. Rev. Lett. 35 (1975) 584

19 Cargill G.S. III, J. Non-Cryst. Solids 61\&62 (1984) 261

20 Blau W., Zschech E. and Bergmann J., Nucl. Inst. and Methods A 261 (1987) 166

21 Davoli I, Stizza S., Paris E., Gargano A. and Seifert F., in X-Ray Absorption fine structure, ed. by S.Samar Hasnain, Ellis Horwood pub., Chichester-England (1991) 315

22 Dalba G., Fornasini P., Rocca F., Lagarde P. and Vlaic G., J. Non-Cryst. Solids 106 (1988) 181

23 Krogh-Moe J., J. Non-Cryst. Solids 1 (1969) 269

24 Feller S.A., Dell W.J. and Bray P.J., J. Non-Cryst. Solids 51 (1982) 21

25 Greaves G.N., in Proc. of the "II XAFS and Near Edge Structures Conference", Springer Series in Chem. Phys. 27 (1983) 248

26 Bernieri E., Burattini B., Dalba G., Fornasini P. and Rocca F., Solid St. Comm. 48 (1983) 421

27 Dalba G., Fornasini P., Rocca F., Bernieri E., Burattini E. and Mobilio S., J. Non-Cryst. Solids $91(1987) 153$

28 Czyzyk M.T., de Groot R.A., Dalba G., Fornasini P., Kisiel A., Rocca F. and Burattini E., Phys. Rev. B 39 (1989) 9831

29 Cervinka L., Rocca F., Fornasini P. and Dalba G., J. Non-Cryst. Solids (1992), in print

30 Mastelaro V., Benazeth S., Dexpert H., Ibanez A. and Ollitrault-Fichet R., J. Non-Cryst. Solids (1992) to be published; Mastelaro V., Dexpert H. and Benazeth S. , in Proc. "VII Int. Conf. on the Physics of Non-Crystalline Solids", Cambridge - England (1991)

31 Inman J.M., Houde-Walter S.N., Greaves G.N., Dobson B.R. and Dent A.J., as reported in Daresbyry Lab. preprint DL/SCI/P772E (1991)

32 Melman H. and Garofalini S.H., J. Non-Cryst. Solids 134 (1991) 107

33 Dalba G., Fornasini P., Rocca F., Burattini E. and Fontana A., Solid State Ionics 28-30 (1988) 713;

34 Rocca F., Dalba G., Fornasini P. and Tomasi A., Solid State Ionics (1992) in print

35 Tatsumisago M., Shinkuma Y. and Minami T, Nature 354 (1991) 217

36 McGreavy R.L. and Pusztai L., Mol. Simul. 1 (1988) 359; Gurman S.J. and McGreavy R.L., J. Phys: Condens. Matter 2 (1990) 9463 